\title{
Reachable set estimation for fuzzy cellular neural networks with bounded disturbances
}

\author{
Shi-li Shao ${ }^{\mathrm{a}}$, He Zhang ${ }^{\mathrm{b},{ }^{*}}$ and Chang-Qing Liu ${ }^{\mathrm{b}}$ \\ ${ }^{a}$ Public course teaching Department, XinXiang vocational and technical college, \\ Xinxiang, 453006, China \\ ${ }^{b}$ Institute of Mathematics and Statistics, Baise University, Baise, 533000, PR China, \\ E-mail: shaoshili1230@sohu.com,freeskyvip@163.com \\ http://www.xxvtc.com \\ http://www.bsuc.cn \\ *Corresponding author
}

\begin{abstract}
This paper is concerned with the problem of reachable set estimation for fuzzy cellular neural network with unit-peak bounded. Based on the Lyapunov-Krasovskii functional approach, delay -dependent conditions for estimating the reachable set of the considered system are derived. Moreover, an example is given to demonstrate the advantages of our method.
\end{abstract}

Keywords: FCNN; Bounded; Reachable Set; Time Delay.

\section{Introduction}

In the past decades, cellular neural network, as a special case of artificial neural networks, and their dynamical characteristics have been extensively investigated. There are numerous excellent results related to the subject have been generated due to its wide application in various fields such as image processing, solving partial differential equations, reducing non-visual problems to geometric maps. Consider the fact that uncertainty or vagueness is inevitable in many practical systems, fuzzy theory is devoted to deal with vagueness. Since fuzzy cellular neural network (FCNN) was proposed by [1]. Therefore, the research of fuzzy cellular neural network has attracted an increasing number of attention and research results have been present by many investigators.

The exponential stability problem of delayed fuzzy cellular neural networks with constant and time-varying delays was considered in [2]. With respect to white blood cell detection, [3] proposed A new detection algorithm based on fuzzy cellular neural networks. [4] dealt with the exponential stability problem of delayed fuzzy cellular neural networks with diffusion. [5] studied the global 
asymptotic stability problem of fuzzy cellular neural networks with time delay in the leakage term under impulsive perturbations, some sufficient conditions were present to guarantee global asymptotic stability of the equilibrium point. [6] and [8] studied the global asymptotic stability problem of a class of bidirectional associative memory delayed fuzzy cellular neural networks. [7] investigated the global exponential synchronization problem of delayed fuzzy cellular neural networks.

On the other hand, the problem of reachable set estimation has received considerable attention. The objective of this subject is to construct a bounding ellipsoid that contains all the reachable states of the considered system under zero conditions. Moreover, there is no doubt that the study of time-delay systems in the field of research is vital part of it. Thus, a number of researchers have devoted their efforts to the issue of reachable set estimation for time-delay systems. As a consequence, many works have been devoted to the issue of reachable set estimation for time-delay systems and its related fields, see [9-17]. the reachable set estimation problem was concerned in [9] for discrete-time linear systems with multiple constant delays and bounded peak inputs. [10-11] concerned with the problem of reachable set estimation for a class of linear systems in the presence of both discrete and distributed delays. Furthermore, the problem of reachable set estimation for a class of delayed neural networks suffered by polytopic uncertainties was addressed in [12].

To the best of our knowledge, the reachable set estimation problem for FCNN with delays has not yet been fully investigated, which is still open. Based on the above discussion, our objective in this paper is to analyze FCNN and presents some sufficient conditions to solve this problem. Moreover, an example is given to demonstrate the advantages of our method.

\section{Preliminaries}

Let $C([-\tau, 0], R)$ be the Banach space of continuous functions which map $[-\tau, 0]$ into $R^{n}$ with the topology of uniform convergence. Consider the following FCNN with delays

$$
\left\{\begin{array}{cc}
\dot{x}_{i}(t)=-a_{i} x_{i}(t)+\bigwedge_{j=1}^{n} \alpha_{i j} f_{j}\left(x_{j}(t-\tau)\right)+{\underset{j=1}{\vee} \beta_{i j} f_{j}\left(x_{j}(t-\tau)\right)}^{n}+x_{j=1}^{n} T_{i j} u_{j}+{\underset{j=1}{\vee}}^{\vee} H_{i j} u_{j}, & t \geq 0, \\
& -\tau<t<0 .
\end{array}\right.
$$

Where $\alpha_{i j}, \beta_{i j}, T_{i j}$ and $H_{i j}$ are elements of fuzzy feedback MIN template, fuzzy feedback MAX template, fuzzy feedforward MIN template and fuzzy 
feedforward MAX template, respectively. $\wedge$ and $\vee$ denote the fuzzy AND and fuzzy OR operation, respectively. $x_{i}, u_{i}$ denote state and input of the $i$ th neuron, respectively. $f_{i}(\square)$ is the activation function, $i, j=1,2, \cdots, n$. $\omega_{i}(t) \in R$ is called to be unit-peak bounded if it satisfies $\left|\omega_{i}(s)\right|^{2} \leq 1$ for all $t \geq 0$. The initial data $\phi_{i}(t)$ is a $C\left([-\tau, 0], R^{n}\right)-$ valued variable. Assume that system (2.1) has a unique global solution on $t \geq 0$, which is denoted by $x(t)$, where $x(t)=\left[x_{1}(t), x_{2}(t), \cdots, x_{n}(t)\right]^{T}$.

The reachable sets of system (1) with unit-peak bounded disturbance inputs are defined by $R_{u p}=\left\{\left.x(t)|x(t), \omega(t) \operatorname{satisfy}(2.1),| \omega(t)\right|^{2} \leq 1\right\}$ and the ellipsoid to be determined is defined by $\varepsilon(P)=\left\{\xi \in R^{n} \mid \xi^{T} P \xi \leq 1\right\}$, where $P>0$ is a real constant matrix. As this is an estimation problem, we want the ellipsoid $\varepsilon(P)$ to be as small as possible. This purpose can be achieved by maximizing a positive scalar $\delta$ subject to

$$
P \geq \delta I>0 \text {. }
$$

Assume that the nonlinear functions $f_{j}(u)$ satisfies the following condition (H1) There are positive constants $L_{i}(i=1,2, \cdots, n)$, such that $\left|f_{i}(u)-f_{i}(v)\right| \leq L_{i}|u-v|$ for any $u, v \in R$.

(H2) For $i=1,2, \cdots, n$, the parameters of system (1) satisfy $-a_{i}+\sum_{j=1}^{n}\left(\left|\alpha_{i j}\right|+\left|\beta_{i j}\right|\right) L_{j}<0$.

Lemma 1. Suppose $x=\left(x_{1}, x_{2}, \cdots, x_{n}\right)^{T}$ and $y=\left(y_{1}, y_{2}, \cdots, y_{n}\right)^{T}$ are two states of system (2.1), then we have

$$
\begin{aligned}
& \left|\stackrel{n}{\wedge} \alpha_{i j} f_{j}\left(x_{j}\right)-\bigwedge_{j=1}^{n} \alpha_{i j} f_{j}\left(y_{j}\right)\right| \leq \sum_{j=1}^{n} L_{j}\left|\alpha_{i j}\right|\left|x_{j}-y_{j}\right| \\
& \left|\underset{j=1}{\vee} \alpha_{i j} f_{j}\left(x_{j}\right)-{\underset{j=1}{\vee}}_{i j}^{n} \alpha_{i j}\left(y_{j}\right)\right| \leq \sum_{j=1}^{n} L_{j}\left|\beta_{i j}\right|\left|x_{j}-y_{j}\right|
\end{aligned}
$$

Where ${ }^{L_{j}}$ is shown as (H1).

Lemma 2. If (H1) and (H2) hold, and $\omega(t)=0$. Then system (1) has a unique equilibrium point $x^{*}=\left(x_{1}^{*}, x_{2}^{*}, \cdots, x_{n}^{*}\right)^{T}$. 
Thus, system (1) with $\omega(t)=0$ admits an equilibrium point $x^{*}=\left(x_{1}^{*}, x_{2}^{*}, \cdots, x_{n}^{*}\right)^{T}$. Let $y_{i}(t)=x_{i}(t)-x_{i}^{*}, \varphi_{i}(t)=\phi_{i}(t)-x_{i}^{*}$, then system (1) becomes

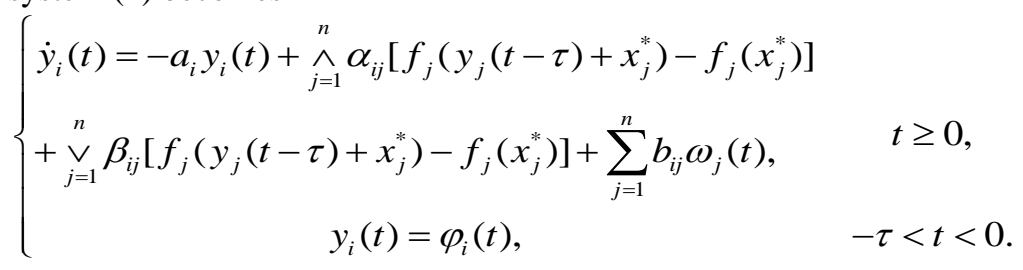

\section{Main Results}

Theorem 1. Consider system (1) with unit-peak bounded disturbance $\omega(t)$. Given a series of scalars $\tau>0, \sigma>0, p_{i}>0$ and $q_{i}>0$ for $i=1,2, \cdots, n$. For $P=\operatorname{diag}\left\{p_{1}, p_{2}, \cdots, p_{n}\right\}$ and $Q=\operatorname{diag}\left\{q_{1}, q_{2}, \cdots, q_{n}\right\}$, if there exist a scalar $\sigma>0$ such that

$$
\Omega=\left(\begin{array}{ccc}
-2 P A+Q & P L & P B \\
* & -Q & O \\
* & * & -\sigma I
\end{array}\right)<0
$$

Then the ellipsoid $\varepsilon(P)$ contains the reachable set $R_{u p}$.

Proof. Taking $V(y(t), t)=e^{-\sigma t} \sum_{i=1}^{n} p_{i} y_{i}(t)^{2}+e^{-\sigma t} \sum_{i=1}^{n} q_{i} \int_{t-\tau}^{t} y_{i}(s)^{2} d s$, we have

$$
\begin{aligned}
& \dot{V}(y(t), t)=-\sigma e^{-\sigma t} \sum_{i=1}^{n} p_{i} y_{i}(t)^{2}+e^{-\sigma t} \sum_{i=1}^{n} p_{i} \dot{y}_{i}(t) y_{i}(t) \\
& -\sigma e^{-\sigma t} \sum_{i=1}^{n} q_{i} \int_{t-\tau}^{t} y_{i}(s)^{2} d s+e^{-\sigma t} \sum_{i=1}^{n} q_{i}\left[y_{i}(t)^{2}-y_{i}(t-\tau)^{2}\right] \\
& \leq-\sigma V(y(t), t)+2 e^{-\sigma t} \sum_{i=1}^{n} p_{i}\left|y_{i}(t)\right|\left\{-a_{i}\left|y_{i}(t)\right|+\hat{j}_{j=1}^{n} \mid \alpha_{i j} \| f_{j}\left(y_{j}(t-\tau)+x_{j}^{*}\right)\right.
\end{aligned}
$$




$$
\begin{aligned}
& \left.-f_{j}\left(x_{j}^{*}\right)\left|+{\underset{j=1}{n}}_{i=1}^{n}\right| \beta_{i j}|| f_{j}\left(y_{j}(t-\tau)+x_{j}^{*}\right)-f_{j}\left(x_{j}^{*}\right)\left|+\sum_{j=1}^{n}\right| b_{i j} \omega_{j}(t) \mid\right\} \\
& +e^{-\sigma t} \sum_{i=1}^{n} q_{i}\left[y_{i}(t)^{2}-y_{i}(t-\tau)^{2}\right] \\
& \leq-\sigma V(y(t), t)+2 e^{-\sigma t} \sum_{i=1}^{n} p_{i}\left|y_{i}(t)\right|\left\{-a_{i}\left|y_{i}(t)\right|+\sum_{j=1}^{n} L_{j}\left|\alpha_{i j}\right|\left|y_{j}(t-\tau)\right|\right. \\
& \left.\sum_{j=1}^{n} L_{j}\left|\beta_{i j}\right|\left|y_{j}(t-\tau)\right|+\sum_{j=1}^{n}\left|b_{i j} \omega_{j}(t)\right|\right\}+e^{-\sigma t} \sum_{i=1}^{n} q_{i}\left[y_{i}(t)^{2}-y_{i}(t-\tau)^{2}\right] \\
& \leq-\sigma V(y(t), t)+2 e^{-\sigma t} \sum_{i=1}^{n} p_{i}\left|y_{i}(t)\right|\left\{-a_{i}\left|y_{i}(t)\right|+\sum_{j=1}^{n} L_{j}\left|\alpha_{i j}\right|\left|y_{j}(t-\tau)\right|\right. \\
& \left.\sum_{j=1}^{n} L_{j}\left|\beta_{i j}\right|\left|y_{j}(t-\tau)\right|+\sum_{j=1}^{n}\left|b_{i j} \omega_{j}(t)\right|\right\}+e^{-\sigma t} \sum_{i=1}^{n} q_{i}\left[y_{i}(t)^{2}-y_{i}(t-\tau)^{2}\right] \\
& +\left(\sigma-\sigma e^{-\sigma t}\right) \omega(t)^{2}
\end{aligned}
$$

Then, we can get

$\dot{V}(y(t), t)+\sigma V(y(t), t)-\sigma \omega(t)^{2} \leq Z \Omega Z^{T} \leq 0$

in which

$Z=\left(\left|y_{i}(t)\right|\left|y_{i}(t-\tau)\right|\left|\omega_{i}(t)\right|\right)$.

Then, by utilizing Lemma 4 in [16], we have $V(y(t), t) \leq 1$ under zero initial conditions. This further implies that $y(t) P y(t)^{T} \leq 1$ since $V(y(t), t) \geq y(t) P y(t)^{T}$. Therefore, the reachable set $R_{u p}$ is contained in $\varepsilon(P)$.

Remark. Theorem 1 gives a sufficient condition for the estimation of the reachable set $R_{u p}$. We can obtain an ellipsoid $\varepsilon(P)$ with the shortest major principal axis by maximizing the positive scalar $\delta$ with respect to the conditions in Eqs. (2) and (4). It should be pointed out that the inequality $\Omega<0$ becomes an LMI when the scalar $\sigma$ is fixed. As shown in [14-15], we can find a local optimum value of $\sigma$ such that the scalar $\sigma$ with respect to condition (2) and (4) is maximized.

\section{Numerical examples}

In this section, we provide an example to show the effectiveness of the result in Theorem 1 on the reachable set estimation problem for system (1) with unitpeak bounded disturbance inputs. To this end, we consider system (1) with 
parameters given by $\quad \omega_{j}=\frac{1}{1+n}$ $f_{1}\left(x_{1}\right)=\frac{1}{2}\left(\left|x_{1}+1\right|+\left|x_{1}-1\right|\right), f_{2}\left(x_{2}\right)=x_{2}, i, j=1,2$. Obviously, $L_{1}=L_{2}=1, \tau=1.2$. Taking $a_{1}=0.75, a_{2}=0.9, \alpha_{11}=0.25$, $\alpha_{12}=0.15, \alpha_{21}=0.25, \alpha_{22}=0.35, \beta_{11}=0.2, \beta_{12}=0.25$, $\beta_{21}=0.15, \beta_{22}=0.25, T_{11}=0.2, T_{12}=0.15, T_{21}=0.15, T_{22}=0.25$, $H_{11}=0.15, H_{12}=0.1, H_{21}=0.2, u_{1}(t)=\cos t, u_{2}(t)=\sin t$. By simple calculation, we can get that there exists $\sigma=1$ such that $\delta_{\max }=2.4335$ and $P=\left[\begin{array}{cc}2.4336 & 0 \\ 0 & 2.433\end{array}\right.$. The simulations of the obtained bounding ellipsoid is shown in Fig. 1.

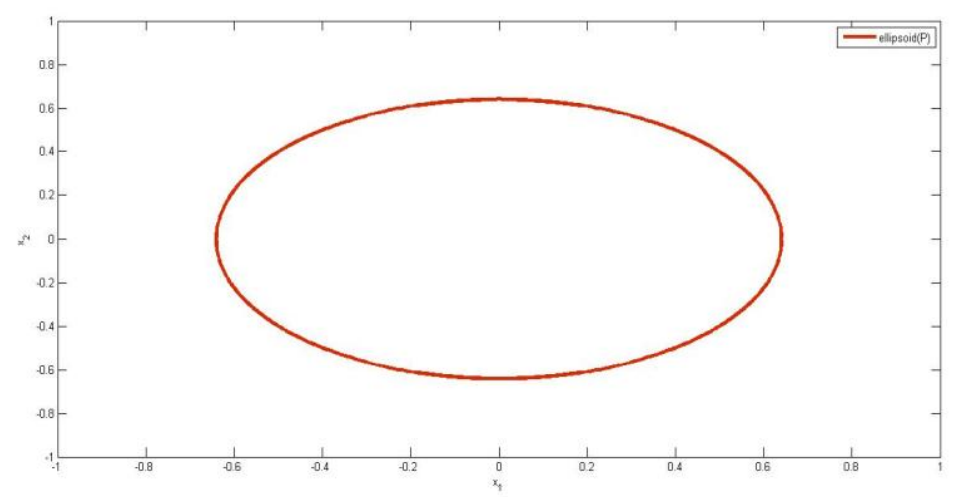

Fig. 1 Bounding ellipsoid for system (2.1)

\section{Acknowledgments}

This research was supported by Middle-aged and young teachers in colleges and universities in Guangxi basic ability promotion project under Grant KX2016LX347, Project in Guangxi department of education under Grant KY2015YB280 and 2014KB03. 


\section{References}

1. Yang, T., Yang, L. B., Wu, C. W., \& Chua, L. O. "Fuzzy cellular neural networks: theory." Cellular Neural Networks and their Applications, 1996. CNNA-96. Proceedings., 1996 Fourth IEEE International Workshop on. IEEE, 1996.

2. Liu, Y., \& Tang, W. "Exponential stability of fuzzy cellular neural networks with constant and time-varying delays." Physics Letters A 323.3 (2004): 224-233.

3. Shitong, W., \& Min, W. "A new detection algorithm (NDA) based on fuzzy cellular neural networks for white blood cell detection." IEEE Transactions on information technology in biomedicine 10.1 (2006): 5-10.

4. Huang, T. "Exponential stability of delayed fuzzy cellular neural networks with diffusion." Chaos, Solitons \& Fractals 31.3 (2007): 658-664.

5. Li, X., Rakkiyappan, R., \& Balasubramaniam, P. "Existence and global stability analysis of equilibrium of fuzzy cellular neural networks with time delay in the leakage term under impulsive perturbations." Journal of the Franklin Institute 348.2 (2011): 135-155.

6. Balasubramaniam, P., Kalpana, M., \& Rakkiyappan, R."Global asymptotic stability of BAM fuzzy cellular neural networks with time delay in the leakage term, discrete and unbounded distributed delays." Mathematical and Computer Modelling 53.5 (2011): 839-853.

7. Feng, X., Zhang, F., \& Wang, W. "Global exponential synchronization of delayed fuzzy cellular neural networks with impulsive effects." Chaos, Solitons \& Fractals 44.1 (2011): 9-16.

8. Li, K. "Impulsive effect on global exponential stability of BAM fuzzy cellular neural networks with time-varying delays." International Journal of Systems Science 41.2 (2010): 131-142.

9. Lam, J., Zhang, B., Chen, Y., \& Xu, S. "Reachable set estimation for discrete-time linear systems with time delays." International Journal of Robust and Nonlinear Control 25.2 (2015): 269-281.

10. Zuo, Z., Ho, D. W. C., \& Wang, Y. "[Brief Paper] Reachable set estimation for linear systems in the presence of both discrete and distributed delays." IET control theory \& applications 5.15 (2011): 1808-1812.

11. Zuo, Z., Fu, Y., \& Wang, Y. "Brief paper: results on reachable set estimation for linear systems with both discrete and distributed delays." IET Control Theory \& Applications 6.14 (2012): 2346-2350.

12. Zuo, Z., Wang, Z., Chen, Y., \& Wang, Y. "A non-ellipsoidal reachable set estimation for uncertain neural networks with time-varying delay." 
Communications in Nonlinear Science and Numerical Simulation 19.4 (2014): 1097-1106.

13. Feng, Z., \& Lam, J. "An improved result on reachable set estimation and synthesis of time-delay systems." Applied Mathematics and Computation 249 (2014): 89-97.

14. Feng, Z., \& Lam, J. "On reachable set estimation of singular systems." Automatica 52 (2015): 146-153.

15. Zuo, Z., Fu, Y., Chen, Y., \& Wang, Y. "A new method of reachable set estimation for time delay systems with polytopic uncertainties." Applied Mathematics and Computation 221 (2013): 639-647.

16. Zuo, Z., Chen, Y., Wang, Y., \& Chen, M. Z. "New criteria of reachable set estimation for time delay systems subject to polytopic uncertainties." IFAC Proceedings Volumes 45.13 (2012): 231-235.

17. Zhang, B., Lam, J., \& Xu, S. "Reachable set estimation and controller design for distributed delay systems with bounded disturbances." Journal of the Franklin Institute 351.6 (2014): 3068-3088. 